Rev Soc Esp Dolor

2014; 21(5): 294

\section{¿En qué basamos nuestras decisiones clínicas?}

\section{Sr. Director:}

Esta última década se ha caracterizado por un incremento exponencial de la información biomédica disponible, lo que puede ser beneficioso o perjudicial en el momento de tomar una decisión clínica. Lo mencionado anteriormente exige que los clínicos, estudiantes e investigadores desarrollen ciertas "habilidades" en el momento de leer un artículo científico y más aún si pretenden basar su decisión clínica en un estudio particular.

Antes de comenzar quisiera plantear la siguiente interrogante, ¿en qué basamos nuestras decisiones clínicas?

De forma "general", la incertidumbre planteada anteriormente se puede responder de muchas formas, entre ellas encontramos:

"Mi decisión clínica se basa en lo que he aprendido en cursos, congresos, universidad (pre-grado/post-grado), experiencia clínica, revistas de especialidad a las que estoy suscrito(a), opinión de expertos en la materia, guías, normas, conversaciones de pasillo con colegas e internet (motores de búsqueda, bases de datos, meta-buscadores, etc.).

Con la introducción de la "Medicina basada en evidencia (MBE)" o "Salud basada en evidencia (SBE)", en los últimos años se ha sobrevalorado la información proveniente de artículos científicos, los cuales están siendo extrapolados a la realidad clínica sin ninguna precaución metodológica (lo que perfectamente puede llevar a una sobre-estimación o sub-estimación del valor real de la intervención). Lo más grave es que han olvidado el sentido de la SBE, la cual se define como: "El uso explícito, racional y juicioso de la mejor evidencia disponible en la toma de decisiones clínicas, incorporando los valores y las preferencias de los pacientes" (1).

Debemos considerar que actualmente la SBE se estructura sobre 3 pilares fundamentales:

1. Experiencia clínica.

2. Evidencia científica.

3. Valores y preferencias de los pacientes.

La experiencia clínica se define como el "dominio creciente del conocimiento y juicio que cada clínico obtiene a través de su práctica diaria (2)". Por el otro lado, la evidencia científica es un pilar fundamental en la formación de cualquier profesional de la salud, debido a que otorga los fundamentos científicos "sólidos" a la experiencia clínica; es decir, aporta un mayor grado de "confianzal certeza" al momento de tomar una decisión clínica (el objetivo de este punto es orientar al clínico para que pueda utilizar la "mejor" información científica frente a una incertidumbre clínica) (3). Los valores y preferencias de los pacientes corresponden a las "expectativas y perspectivas" que estos adquieren en el momento de recibir una intervención (no debemos olvidar que lo más importante de este proceso es respetar a nuestros pacientes, considerando no tan sólo el riesgo-beneficio o coste-efectividad de la intervención, sino que también debemos tener presente las creencias, cultura, etc. de los mismos) (4,5).

No debemos olvidar que en el momento de tomar una decisión clínica tenemos que considerar la experiencia del clínico, la evidencia actual y los valores y preferencias del paciente; por tal motivo la decisión de cuándo y a quién aplicar la evidencia será de exclusiva responsabilidad del clínico.

R. Aguilera Eguía

Facultad de Ciencias de la Salud. Carrera de Fonoaudiología. Universidad de las Américas. Kinesiología. Red Chilena de Salud Basada en Evidencia. Musculoskeletal Group Cochrane Collaboration. Member of the Cochrane Trainer's Network. Member of the Grade Working Group. Chile

\section{BIBLIOGRAFÍA}

1. Sackett D, Rosenberg W, Gray J, et al. Evidence based medicine: what it is and what it isn't. BMJ 1996;312:71-2.

2. Medicina basada en la evidencia. En: conferencia medicina basada en la evidencia. Bonfill X. microcine de la Facultad de ciencias médicas de la unlP; 1998.

3. Guerra 1. La medicina basada en la evidencia: un intento de acercar la ciencia al arte de la práctica clínica. Med Clin (Barc) 1996;107:377-82.

4. Evidence Based Medicine Working Group. Evidence based medicine. A new approach to teaching and practice medicine. JAMA 1992;268:2420-514.

5. Guyatt G, Rennier D. User's guide to the medical literature, a manual for evidence based clinical practice. Chicago, IL: American Medical Association; 2002. 